\title{
Intraoperative Radiation Therapy for Breast Cancer: Are We There Yet?
}

\author{
Chirag Shah, MD \\ Department of Radiation Oncology, Taussig Cancer Institute, Cleveland Clinic, Cleveland, OH
}

The role of Intraoperative radiation therapy (IORT) for breast cancer remains a controversial topic. While representing an advance in treatment efficiency and patient convenience, concerns have existed regarding long-term outcomes, with initial studies demonstrating increased local recurrence (LR) rates, as well as concerns regarding how the technique compared with traditional adjuvant radiation therapy (RT) approaches. ${ }^{1}$ IORT can be delivered with multiple approaches, with the most common being low-energy IORT and electron IORT. Recent updates to IORT data are now available and have raised the question of whether IORT is a standard approach in patients with low-risk early stage breast cancer following breast conserving surgery (BCS).

Low-energy IORT has been evaluated by the TARGITA randomized trial; the study initially included 3451 women randomized to IORT or whole breast irradiation (WBI) with $15 \%$ of all IORT patients receiving additional WBI. Of note, the study included 2 groups, those receiving IORT at the time of surgery (pre-pathology) and those that received IORT as a second procedure (post-pathology). The trial was previously published with 5-year outcomes (29-month follow-up) demonstrating an increase in LR with IORT (3.3\% IORT vs. $1.3 \% \mathrm{WBI}$, though within the non-inferiority criteria) for the entire study population; the post-pathology cohort had an increased rate of LR with IORT (5.4\% IORT vs. $1.7 \% \mathrm{WBI}$, exceeding the non-inferiority criteria) as did the pre-pathology cohort $(2.1 \%$

(C) Society of Surgical Oncology 2020

First Received: 28 September 2020

Accepted: 28 October 2020;

Published Online: 18 November 2020

C. Shah, MD

e-mail: shahc4@ccf.org
IORT vs. $1.1 \%$ WBI, within the non-inferiority criteria). ${ }^{2}$ Recently, updates from TARGIT-A were published; however, surprisingly, the entire study population was not published together so data is only available for the pre- and post-pathology cohorts separately. This raises a concern, as Dr. Cuzick (former chairman of the data monitoring committee for TARGIT-A) noted in 2014, "Another common but well known danger is to focus attention on the most favourable subgroup. The protocol clearly states that the primary analysis population includes all randomised patients." ${ }^{3}$ As such, the updated analyses do not meet this standard and should not be revised after the fact. With further follow-up, the post-pathology cohort (1153 patients) was found to have increased LR with IORT at 5 years $(3.96 \%$ IORT vs. $1.05 \% \mathrm{WBI})$, exceeding the noninferiority threshold and supporting the conclusion that this approach should not be offered to patients at this time. ${ }^{4}$ With respect to the pre-pathology group, LR was elevated with IORT at 5 years $(2.11 \%$ IORT vs. $0.95 \% \mathrm{WBI})$, though within the non-inferiority criteria; long-term Kaplan-Meier curves for LR were not presented by the investigators. ${ }^{5}$ However, significant concerns have been raised regarding the methodology of these updates; the study did not present long-term absolute LR rates but rather local recurrence-free survival (LRFS). This is important because concerns exist regarding composite endpoints such as LRFS which includes deaths; additionally, other partial breast or breast radiotherapy trials have presented longterm LR, which is an important outcome when counseling patients on radiation therapy approaches. ${ }^{6}$ When evaluating LRFS events in the pre-pathology updated data (167 IORT vs. $147 \mathrm{WBI}$ ) and removing deaths (110 IORT vs. $131 \mathrm{WBI})$, it appears there is a large absolute difference in local events (57 IORT vs. 16 WBI from manuscript, 60 LR IORT vs. $24 \mathrm{WBI}$ from Table 3 in updated pre-pathology manuscript), though it is unclear what the absolute LR rates 
are. Additionally, long-term LR rates were not broken down for pre-pathology patients who received TARGIT only, compared with those that received TARGIT and WBI (roughly $20 \%$ of the pre-pathology population); this is essential for informing patients about their long-term absolute local recurrence risks by approach. Additionally, further concern exists, as the recently updated TARGIT-R [a multi-institutional retrospective registry from 19 institutions in North America treated with IORT alone (79\%) or as a boost $(21 \%, 14 \%$ planned boost)] demonstrated a 5 -year LR rate of $7.6 \%$ for primary IORT with 667 patients included. ${ }^{7}$

With respect to electron IORT, the ELIOT trial was a randomized trial of 1305 women with patients randomized to electron IORT or WBI; unlike TARGIT-A, no additional WBI was given to patients undergoing IORT. At 5 years, increased local recurrences (4.4\% IORT vs. $0.4 \% \mathrm{WBI}$ ) was seen with electron IORT. ${ }^{8}$ Long-term outcomes from ELIOT have yet to be published; however, Leonardi et al. evaluated 1822 patients receiving electron IORT at a single institution outside of a clinical trial and found that those meeting American Society for Radiation Oncology (ASTRO) suitable criteria had low 5-year rates of local recurrence $(1.5 \%)$ with higher rates of recurrence seen in the cautionary $(4.4 \%)$ and unsuitable cohorts $(8.8 \%){ }^{9}$

For clinicians, including breast surgeons and radiation oncologists, the question remains, what is the role of IORT for patients with early stage breast cancer following BCS in 2020? At this time, current guidelines from ASTRO recommend patients to be counseled on increased rates of LR with IORT while the American Brachytherapy Society (ABS) (though published prior to the TARGIT-A updates) do not support IORT outside of prospective studies. For low-energy IORT, ASTRO guidelines note it should only be used within a prospective study (registry or clinical trial) and when used that only suitable patients be considered, while ABS guidelines do not recommend it outside of prospective studies. ${ }^{10},{ }^{11}$ With respect to electron-IORT, the ABS guidelines do not recommend this approach outside of clinical trial, while the ASTRO guidelines note that electron IORT should be restricted to suitable patients. ${ }^{10},{ }^{11}$ It is important to recognize that both guidelines included members with IORT experience. ${ }^{10},{ }^{11}$

At this time, in light of the outcomes available and evidence-based guidelines, IORT for early stage breast cancer remains investigational. As part of shared decision making, clinicians should advise patients regarding concerns with IORT outcomes and that guidelines provide limited support for IORT at this time. Fortunately for patients, while not single fraction, randomized studies have supported the emergence of short-course (5 fraction) whole breast and partial breast techniques with long-term follow- up, providing an evidence based adjuvant radiotherapy option for patients with a shorter duration of treatment than traditional approaches.

\section{FUNDING None.}

DISCLOSURES Chirag Shah, MD - Consultant Impedimed, Consultant PreludeDX. Grants - Varian Medical Systems, VisionRT, PreludeDX.

\section{REFERENCES}

1. Shah C, Wobb J, Khan A. Intraoperative radiation therapy in breast cancer: still not ready for prime time. Ann Surg Oncol. 2016;23:1796-8.

2. Vaidya JS, Wenz F, Bulsara M, et al. Risk-adapted targeted intraoperative radiotherapy versus whole-breast radiotherapy for breast cancer: 5-year results for local control and overall survival from the TARGIT-A randomised trial. Lancet. 2014;383:603-13.

3. Cuzick J. Radiotherapy for breast cancer, the TARGIT-A trial. Lancet. 2014;383:1716.

4. Vaidya JS, Bulsara M, Saunders C, et al. Effect of delayed targeted intraoperative radiotherapy vs whole-breast radiotherapy on local recurrence and survival: long-term results from the TARGIT-A randomized clinical trial in early breast cancer. JAMA Oncol. 2020;6:e200249.

5. Vaidya JS, Bulsara M, Baum M, et al. Long-term survival and local control outcomes from single dose targeted intraoperative radiotherapy during lumpectomy (TARGIT-IORT) for early breast cancer: TARGIT-A andomized clinical trial. BMJ. 2020;370:m2836.

6. Bentzen SM, Haviland JS. No apparent effect of 50-kV intraoperative radiation therapy on loco-regional control after lumpectomy for breast cancer. BMJ. 2020. https://www.bmj.co m/content/370/bmj.m2836/rapid-responses.

7. Valente S, Ashikari A, Bethke K, et al. TARGIT-R (retrospective): Five-year follow-up on Intraoperative Radiation Therapy (IORT) for breast cancer performed in North America. American Society of Breast Surgeons Annual Meeting, May 2020. https:// www.breastsurgeons.org/education/virtual/proceedings.pdf.

8. Veronesi U, Orecchia R, Maisonneuve P, et al. Intraoperative radiotherapy versus external radiotherapy for early breast cancer (ELIOT): a randomized controlled equivalence trial. Lancet Oncol. 2013;14:1269-77.

9. Leonardi MC, Maisonneuve P, Mastropasqua MG, et al. How do the ASTRO consensus statement guidelines for the application of accelerated partial breast irradiation fit intraoperative radiotherapy? A retrospective analysis of patients treated at the European Institute of Oncology. Int $J$ Radiat Oncol Biol Phys. 2012;83:806-13.

10. Correa C, Harris EE, Leonardi MC, et al. Accelerated partial breast irradiation: Executive summary for the update of an ASTRO evidence-based consensus statement. Pract Radiat Oncol. 2017;7:73-9.

11. Shah C, Vicini F, Shaitelman SF, et al. The American Brachytherapy Society consensus statement for accelerated partial-breast irradiation. Brachytherapy. 2018;17:154-70.

Publisher's Note Springer Nature remains neutral with regard to jurisdictional claims in published maps and institutional affiliations. 